\title{
Single Cell RNA Sequencing Reveals Critical Functions of $M k x$ in Periodontal Ligament Homeostasis
}

\author{
Kaho Takada ${ }^{1,2 t}$, Tomoki Chiba ${ }^{1 \dagger}$, Takayuki Miyazaki ${ }^{1,3}$, Lisa Yagasaki ${ }^{1,4}$, Ryo Nakamichi $^{5}$, \\ Takanori Iwata ${ }^{4}$, Keiji Moriyama ${ }^{3}$, Hiroyuki Harada ${ }^{2}$ and Hiroshi Asahara ${ }^{1,5 *}$ \\ ${ }^{1}$ Department of Systems BioMedicine, Graduate School of Medical and Dental Sciences, Tokyo Medical and Dental University, \\ Bunkyo-ku, Japan, ${ }^{2}$ Department of Oral and Maxillofacial Surgery, Graduate School of Medical and Dental Sciences, Tokyo \\ Medical and Dental University, Bunkyo-ku, Japan, ${ }^{3}$ Department of Maxillofacial Orthognathics, Graduate School of Medical and \\ Dental Sciences, Tokyo Medical and Dental University, Bunkyo-ku, Japan, ${ }^{4}$ Department of Periodontology, Graduate School of \\ Medical and Dental Sciences, Tokyo Medical and Dental University, Bunkyo-ku, Japan, ${ }^{5}$ Department of Molecular and \\ Experimental Medicine, The Scripps Research Institute, La Jolla, CA, United States
}

\section{OPEN ACCESS}

Edited by:

Brian Frank Eames,

University of Saskatchewan, Canada

Reviewed by:

Vincent Everts,

VU Amsterdam, Netherlands Andrew Jheon,

University of California, San Francisco, United States

*Correspondence: Hiroshi Asahara asahara.syst@tmd.ac.jp

${ }^{\dagger}$ These authors have contributed equally to this work

Specialty section:

This article was submitted to Molecular and Cellular Pathology, a section of the journal Frontiers in Cell and Developmental Biology

Received: 15 October 2021 Accepted: 14 January 2022 Published: 04 February 2022

Citation:

Takada K, Chiba T, Miyazaki T, Yagasaki L, Nakamichi R, Iwata $T$, Moriyama $K$, Harada $H$ and Asahara $H$ (2022) Single Cell RNA Sequencing Reveals Critical Functions of Mkx in Periodontal Ligament Homeostasis. Front. Cell Dev. Biol. 10:795441. doi: 10.3389/fcell.2022.795441
The periodontal ligament (PDL) comprises a fibrous tissue that connects teeth to alveolar bone and is essential for periodontal function. The transcription factor mohawk homeobox $(M k x)$ is expressed in the PDL where it plays an important role in the development and maintenance of the PDL. However, the precise and critical functions of Mkx in the cell populations comprising PDL have not yet been elucidated. The present study aimed to clarify the effects of a MkX deficiency on PDL cellular heterogeneity and differences between gene expression in PDL tissues from wild-type (WT) $\left(M k x^{+/+}\right)$and $M k x$ knockout $\left(\mathrm{MkX}^{-/-}\right)$rats using single-cell RNA sequencing. We identified 12 cell clusters comprising mesenchymal cells and macrophages. The expression of MkX and scleraxis (Scx; another key transcription factor of PDL), was mutually exclusive, and partitioned mesenchymal cell clusters into Mkx and Scx types that dominantly expressed proteoglycans and elastic fibers, and type 1 and 3 collagen, respectively. Ossificationrelated genes were upregulated in mesenchymal cell and osteoblast clusters with more $M k x^{-/-}$than $M k x^{+/+}$PDLs. Increased number of cells and inflammatory mediators were observed in macrophage clusters of $M k^{-/-}$PDL. These results suggested that $M k x$ plays an important role in maintaining PDL homeostasis by regulating specific cell populations and gene expression.

Keywords: MKX, SCX, periodontal ligament, single cell RNA-sequencing (scRNA-seq), extracellular matrix (ECM), collagen, ossification, inflammation

\section{INTRODUCTION}

The periodontal ligament is a fibrous tissue that connects alveolar bone to the cementum that covers teeth. The periodontal ligament comprises fibroblasts, osteoblasts, blood vessels, nerves, epithelial cells, and a rich extracellular matrix (ECM) (Beertsen et al., 1997). The ECM of the PDL includes principal fibers that comprise mainly type 1 and type 3 collagen, and oxytalan fibers that consist of elastic fibers, proteoglycans, and glycosaminoglycans that are mainly produced by periodontal ligament fibroblasts (Ishikawa et al., 2009; Strydom et al., 2012; McKee et al., 2013; Chen et al., 2021). The fibers in PDL function as cushions to protect the teeth and alveolar bone from physical damage caused by chewing motions of teeth, flow generated by tongue motions, and orthodontic forces 
(Beertsen et al., 1997). In particular, fibroblasts derived from mesenchymal cells are important for maintaining space between the teeth and alveolar bone by producing an ECM in response to mechanical loading (Chukkapalli and Lele, 2018).

The periodontium hosts various oral bacteria, some of which are responsible for periodontitis (Holt and Ebersole, 2005; Lamont and Hajishengallis, 2015). Chronic inflammation induced by oral bacteria leads to periodontitis, systemic inflammation, and metabolic diseases (Socransky and Haffajee, 2005; Lalla and Papapanou., 2011; Kinane et al., 2017; Hajishengallis and Chavakis, 2021). Inflammation of the periodontium induces not only the expression of inflammatory cytokines and chemokines, but also enzymes involved in ECM degradation (Franco et al., 2017; Nilsson, 2021). Mechanical loading of the PDL by orthodontic and occlusal forces also induces the production of ECM and the activation of osteoclasts and osteoblasts (Harrel, 2003). Thus, inflammation induced by oral bacteria and mechanical loading in PDL elicits ECM and bone remodeling, which is important for PDL homeostasis (Lerner, 2006; Chukkapalli and Lele, 2018).

Tendons and ligaments that mainly comprise type 1 collagen, express the transcription factor mohawk homeobox $(M k x)$ that is important for their development and homeostasis (Ito et al., 2010; Liu et al., 2010). We previously showed that $M k x$-deficient mice and rats have hypoplastic Achilles' tendons with endochondral ossification (Ito et al., 2010; Suzuki et al., 2016). $M k x$ is also expressed in the PDL (Koda et al., 2017). Mice deficient in $M k x$ develop an age-related reduction in PDL fibers and increased ossification, indicating that $M k x$ plays an important role in PDL homeostasis (Koda et al., 2017). The transcription factor Scx, which is also important for the development of tendons and ligaments, is expressed in the PDL (Cserjesi et al., 1995; Schweitzer et al., 2001; Takimoto et al., 2015). Knockdown of Scx in periodontal ligament cells result in a significant increase in osteocalcin expression in response to osteogenic differentiation, suggesting that $S c x$ also suppresses PDL ossification (Takimoto et al., 2015). Thus, $M k x$ and Scx might play similar roles in homeostasis by maintaining the ligament-like properties of the PDL.

Transcriptome analysis at the single-cell level is a powerful tool for revealing the diversity and complexity of cells and relationships among genes involved in tissues (Tanay and Regev, 2017). Analyses of dental follicles, dental pulp, and PDL from humans and mice using single-cell RNA-sequencing (scRNA-seq) have generated the following notable results. Three mesenchymal stem cell subclusters have been identified in human dental pulp and periodontium (Pagella et al., 2021). Dental follicle subpopulations expressing parathyroid hormone (PTH)-related peptide (PTHrP) are important for tooth eruption via the PTH/ PTHrP receptor axis (Takahashi et al., 2019). The heterogeneity of epithelial cells and their roles in the incisors of neonatal mice have been clarified (Chiba et al., 2020). Cell subtypes between human and mouse teeth differ at the molecular level and are species-specific (Krivanek et al., 2020). However, the relationships between $M k x$ and $S c x$ and their role(s) in PDL remain unknown.
Here, we performed single-cell RNA sequencing (scRNA-Seq) of periodontal ligament derived cells from $M k x$ knockout $\left(M k x^{-/-}\right)$and WT rats to reveal the critical function of $M k x$ in periodontal ligament tissues. Our experiment benefits from the natural advantage of the $M k x$ knockout rats in providing a sufficient number of cells in comparison to the conventional knockout mouse.

\section{MATERIALS AND METHODS}

\section{Animals}

$M k x$-deficient rats were generated by deleting nucleotides downstream of the start codon in the second exon using CRISPR/Cas9, as reported previously (Suzuki et al., 2016). Wild type Wistar rats were obtained from Sankyo Lab Service (Tokyo, Japan). All animals used in this study were 6 weeks-old male rats. All animal experiments were performed in accordance with the protocols approved by the Institutional Animal Care and Use Committee of the Tokyo Medical and Dental University (approval no. A2021-064A).

\section{Isolation of Periodontal Ligament Cells}

Twelve upper, lower, left, and right molars extracted from two $\mathrm{Mkx}^{+/+}$and $\mathrm{Mkx}^{-/-}$rats were dissociated in serum-free Dulbecco modified Eagle medium (Sigma-Aldrich Corp., St. Louis, MO, United States) containing collagenase $(2 \mathrm{mg} / \mathrm{ml})$ and $0.25 \%(\mathrm{w} / \mathrm{v})$ trypsin (both from Wako Pure Chemical Industries, Osaka, Japan) for $1 \mathrm{~h}$ at $37^{\circ} \mathrm{C}$ using a Thermomixer at $1,200 \mathrm{rpm}$ (Eppendorf, Hamburg, Germany). Dissociated PDLs were passed through a $40-\mu \mathrm{m}$ nylon cell strainer to remove debris, centrifuged at $800 \times g$ for $5 \mathrm{~min}$, then suspended in FACS buffer (phosphate-buffered saline [PBS] containing 1\% penicillin/ streptomycin (Wako Pure Chemical Industries) and 10\% FBS (Thermo Fisher Inc., Waltham, MA, United States). The cells were then stained with $2 \mu \mathrm{g} / \mathrm{ml}$ of propidium iodide (BioLegend, San Diego, CA, United States) and $1 \mu \mathrm{g} / \mathrm{ml}$ of Hoechst 33342 (Thermo Fisher Scientific Inc.). Live cells that were negative for propidium iodide and positive for Hoechst 33342 were sorted using MoFlo XDP (Beckman Coulter, Inc., Brea, CA, United States).

\section{Single Cell Isolation, Library Preparation and Sequencing}

Gel beads in emulsion (GEM) were generated using Chromium Next GEM Single Cell 3' Library Kit v3 (10x Genomics, Pleasanton, CA, United States) as described by the manufacturer. Briefly, 24,000 single cells were captured using a Chromium Single Cell A Chip. A library was constructed, then sequenced using a HiSeq $\mathrm{X}$ system or NextSeq1000 system (Illumina Inc., San Diego, CA, United States) with $2 \times 150$ bp reads to produce 400 to 800 million reads per sample.

\section{Data Analysis}

The reference sequence for the rat genome was Rattus norvegicus Rnor_6.0. The $M k x$ gene was not in the annotation file because it 

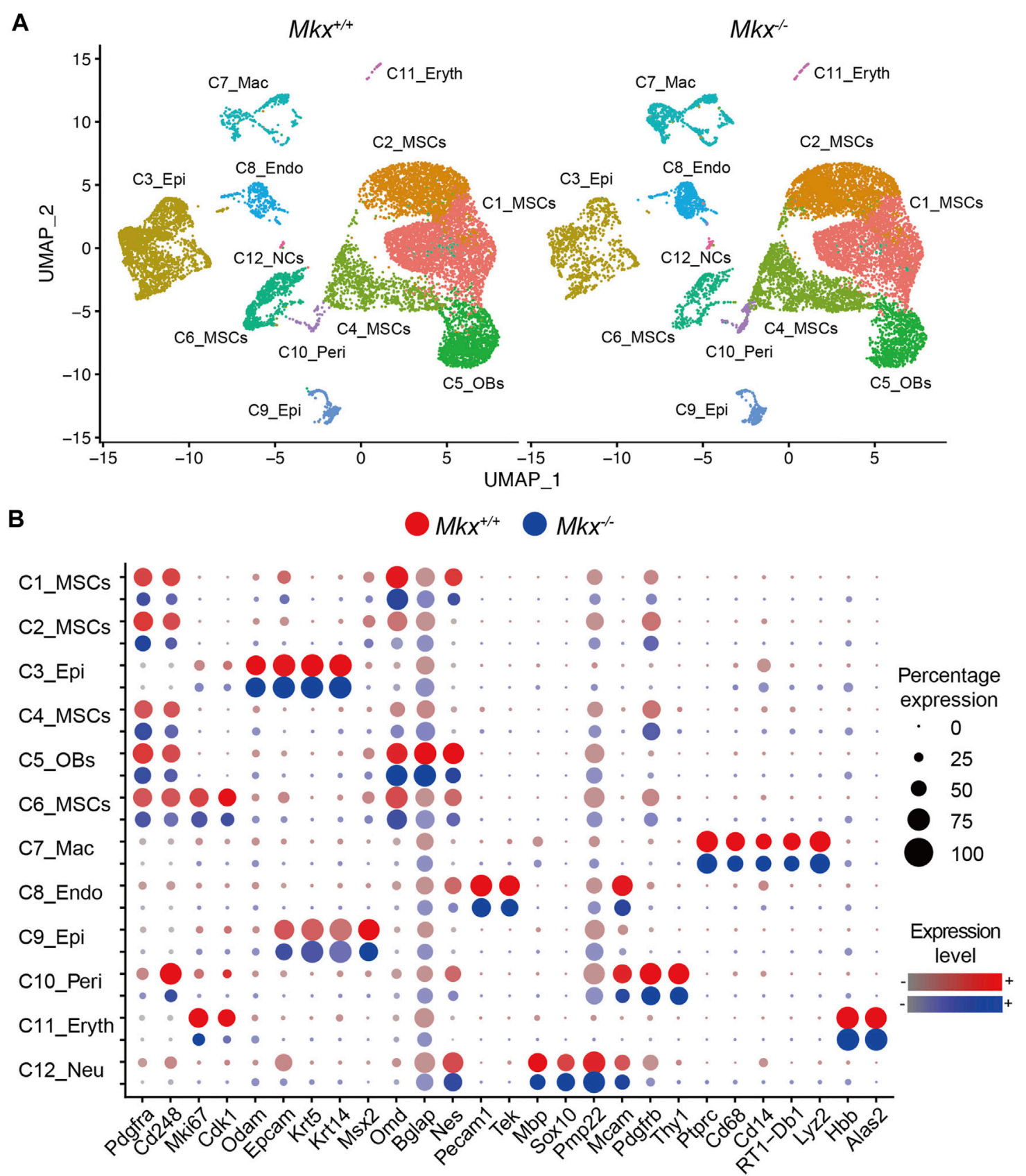

FIGURE 1 | Identification of cell populations in rat PDL. (A) Uniform Manifold Approximation and Projection (UMAP) plot of PDL cells $(n=24,000)$ derived from $\mathrm{MkX}^{+/+}$and $\mathrm{MkX}^{-/-}$rats. (B) Dot plot for markers of cell types. The size of each circle reflects the percentage of cells in a cluster where the gene is detected, and the color intensity reflects the average expression level within each cluster. 12 different clusters (C) were detected (C1 to C12). Endo, endothelial cells; Epi, epithelial cells; Eryth, erythrocytes; Mac, macrophage; MSCs, mesenchymal stromal cells; NCs, neural cells; OBs, osteoblasts; Peri, pericytes.

is registered as predicted transcript XM_017600733 in the Rnor_6.0 genome. Therefore, we created a new gtf file to which we manually added $M k x$ transcript annotation. Cell Ranger (v. 6.1.1, 10x Genomics) was used to demultiplex samples, process barcodes, and align them with Rnor_6.0. The index file required for alignment was created using cellranger's mkref based on the annotation file for Rnor_6.0, with $M k x$ annotation. Cell barcodes were processed and aligned to the genome by STAR using cellranger's count with the force-cells option. Individual samples were integrated, expression was normalized, and cell populations were clustered based on the matrix files of gene expression generated for each cell using $\mathrm{R} \mathrm{v}$. 4.1.2 (R Foundation for Statistical Computing, Vienna, Austria) and the R package Seurat v. 4.0.6 (Butler et al., 2018) as described by the developer. All $M k x^{+/+}$and $M k x^{-/-}$datasets were analyzed independently then combined for integrated analysis. Genes 
expressed in $<3$ cells, cells with $<2,000$ unique molecular identifiers (UMIs) and $<200$ genes were removed from the gene expression matrix for each dataset. Since mitochondrial genes were not registered in the rat annotation file, the data were not filtered based on mitochondrial gene contamination. The data were log-normalized, and the expression of each gene was scaled by regressing the number of UMIs. The gene expression matrix was assessed using principal component analysis (PCA) and the first 15 principal components were used for clustering and visualization. We applied unsupervised shared nearest neighbor (SNN) clustering to the genes with a resolution of 0.2 and visualized them using Uniform Manifold Approximation and Projection (UMAP). Uniquely expressed genes in each cluster were analyzed using the Seurat FindConservedMarkers function. Differentially expressed genes were considered only if the average expression in the cluster was $\log 2$ fold-change $<-0.5$ and $>1.5$. Individual gene expression was determined using the Seurat FetchData function. Gene ontology was analyzed using Metascape (https://metascape.org/gp/index. $\mathrm{html} /$ /main/step1). Transcription factors involved in differentially expressed genes expression were analyzed by Pscan (http://159.149.160.88/pscan/).

\section{RESULTS}

\section{Periodontal Ligament Mainly Comprises a Cell Population With Features of Mesenchymal Stromal Cells}

We analyzed PDLs derived from WT $\left(M k x^{+/+}\right)$and $M k x^{-/-}$rats using scRNA-Seq and identified cell populations containing pericytes, erythrocytes macrophages, osteoblasts, and mesenchymal, epithelial, vascular endothelial, and neural cells (Figure 1A). Clusters C1, C2, C4, and C6 comprised mesenchymal stromal cells (MSCs) that were CD45 (Ptprc)', Platelet-derived growth factor receptor alpha $(P d g f r a)^{+}$, and $\mathrm{CD} 248(\mathrm{Cd} 248)^{+}$, and were the largest population in the PDL (C1_MSCs, C2_MSCs, C4_MSCs and C6_MSCs). The C6_MSCs were highly proliferative with elevated Ki67 (Mki67) and cyclin A1 (Cdk1) expression. Cluster C5 is considered to be an osteoblast because the following were expressed: Osteomodulin, $(\mathrm{Odm})$, Osteocalcin (Bglap) and Nestin (Nes) as well as $\mathrm{CD}^{-} 5^{-}$, Pdgfra ${ }^{+}$and $\mathrm{CD}_{248^{+}}$(C5_osteoblasts [OBs]). Clusters C3 and C9 were Epcam ${ }^{+}$, keratin $(\mathrm{Krt}) 14^{+}$, and $\mathrm{Krt5} 5^{+}$ epithelial cell clusters and the second largest cell population in the PDL (C3_epithelial (Epi) and C9_Epi). C3_Epi expresses abundant Odontogenic ameloblast-associated protein (Odam), while C9_Epi expresses abundant MSH homeobox 2 (Msx2), suggesting that they are epithelial cell populations with different functions. We also identified CD31 $(\text { Pecam } 1)^{+}$and Tie-2 $(\text {Tek })^{+}$ vascular endothelial cells (C8_Endo), Myelin basic protein $(M b p)^{+}$, Sry-related HMg-Box gene $10(\text { Sox 10 })^{+}$and Peripheral myelin protein $22(P m p 22)^{+}$neural cells, including oligodendrocytes and Schwann cells (C12_NCs), CD146 $(\mathrm{Mcam})^{+}$, Platelet-derived growth factor receptor beta $(P d g f r b)^{+}$and Thy-1 cell surface antigen $(T h y 1)^{+}$pericytes
(C10_Peri). We identified $\mathrm{CD}^{+} 5^{+}, \mathrm{CD} 14(\mathrm{Cd} 14)^{+}, \mathrm{CD} 68$ $(C d 68)^{+}$, MHC class II $(R T 1-D b 1)^{+}$and lysozyme $(L y z 2)^{+}$ macrophages (C7_Mac), as well as hemoglobin beta $(\mathrm{Hbb})^{+}$ and aminolevulinic acid synthase 2, erythroid (Alas2) ${ }^{+}$ erythrocytes (C11_Eryth) (Figure 1B).

\section{Mkx and Scx Are Expressed in Different Cell Clusters}

Figure 1B shows mesenchymal cells divided into four subclusters. The C1_MSC cluster comprised cells in a transitional state expressing markers related to epithelial cells and osteoblasts, and the C6_MSCs cluster were highly proliferative cells. We analyzed the expression of $M k x$ and $S c x$, which are important transcription factors in the formation and maintenance of the periodontal ligament (Takimoto et al., 2015; Koda et al., 2017; Miyazaki et al., 2021). We found that $M k x$ and $S c x$ were expressed in all subclusters (Figure 2A). Cells expressing $M k x$ and $S c x$ were the most abundant in the C2 and C4_MSC clusters, respectively. Since only a few cells expressed both $M k x$ and $S c x$, we considered that their expression was mutually exclusive (Table 1). Furthermore, the numbers of cells in the C4_ MSC cluster with abundant $S c x^{+}$cells, was increased 2 -fold, and the number of $S c x^{+}$cells increased 2.5-fold in clusters from $M k x^{-/-}$PDL, compared with $M k x^{+/+}$PDL (Table 1 and Figure 2B). However, the expression of $S c x$ in this cluster was comparable between $M k x^{+/+}$and $M k x^{-/-}$PDL (Figure 2B). We analyzed genes that were highly expressed in C2_MSCs and C4_MSCs. Periostin (Postn), type I collagen (Colla1 and Colla2), type III collagen (Col3a1), Alkaline phosphatase $(A l p l)$, Decorin $(D c n)$, Tenascin-N $(T n n)$, Collagen beta $(1-\mathrm{O})$ galactosyltransferase 1 (Colgalt1), Lysyl oxidase homolog 2 (Lox2) were identified in the C4_ MSC cluster. Postn, Colgalt1, Loxl2, and Dcn, which are related to the maturation of type 1 and type 3 collagen, were upregulated in the C4_ MSC cluster (Figure 2C). In contrast, the expression of Martlilin-4 (Matn4), Microfibrillar-associated protein 4 (Mfap4), and Fibulin-1 $(F b \ln 1)$, which are involved in the formation of oxytalan fibers, was high in C2_MSCs. Proteoglycans such as Versican (Vcan), Syndecan-1 (Sdc1), and Collagen alpha-2(IX) chain (Col9a2), which interact with proteoglycans, were significantly more abundant in C2_MSCs (Figure 2D). These results suggested that cells expressing $S c x$ are relatively abundant among populations that produce collagen, whereas those expressing $M k x$ are found among populations that produce oxytalan fibers and proteoglycan.

\section{$\mathrm{Mkx}^{-/-}$PDL Mesenchymal Cell Clusters Have Ossification Gene Expression Profiles} Differentially expressed genes between $M k x^{+/+}$and $M k x^{-/-}$PDL were analyzed in the C2_ MSC cluster, which contained a lot of cells expressing Mkx. 405 genes were upregulated (Figure 3A). Gene ontology analysis revealed that many genes were involved in connective tissue development $(\log p=-6.19)$ and skeletal system development $(\log p=-5.04)$. The expression of parathyroid hormone like hormone (Pthlh), Insulin Like Growth Factor 

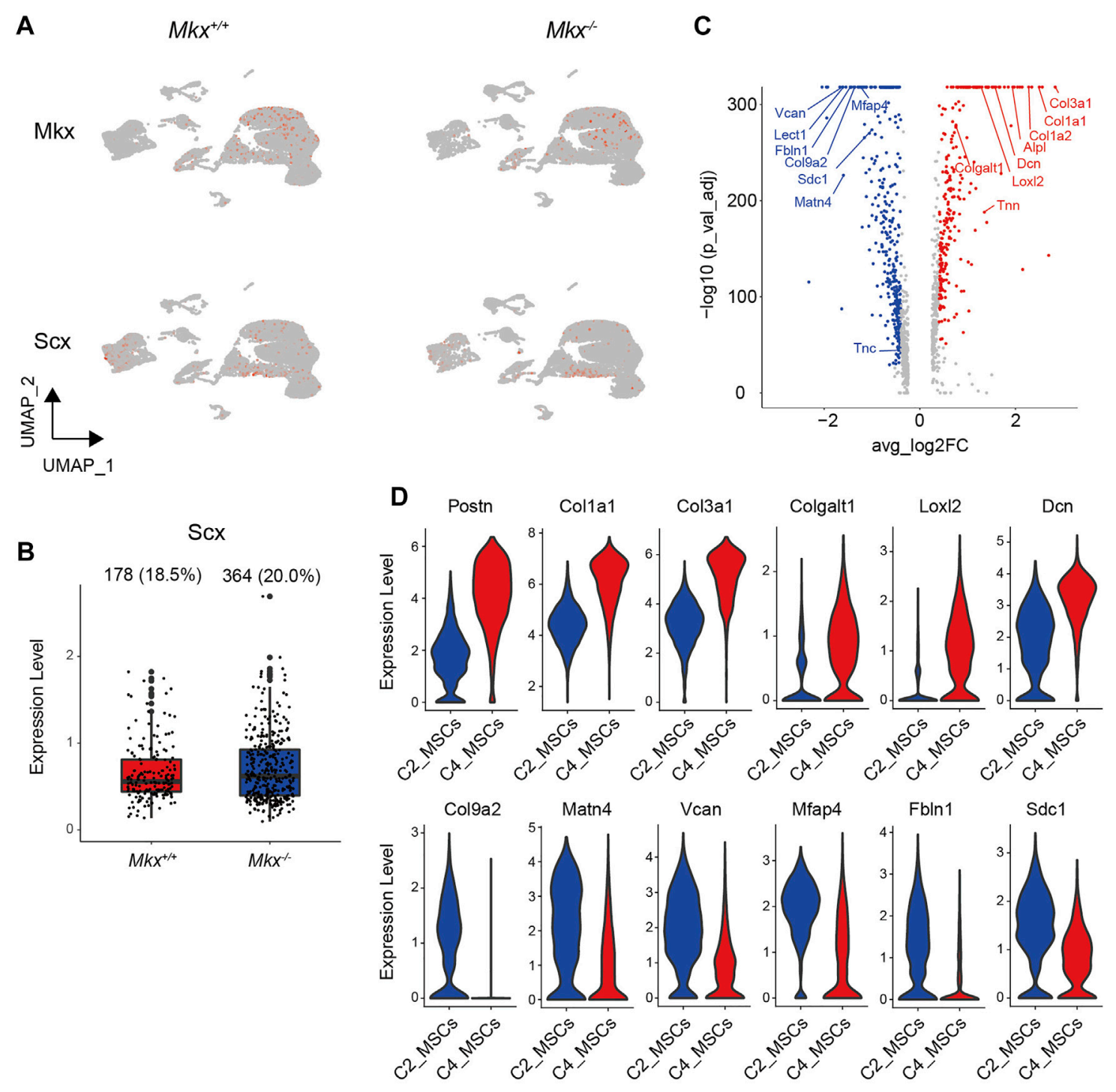

FIGURE 2 |Mkx and Scx are expressed in different cell clusters. (A) UMAP plot of Mkx and Scx expression in cell clusters. Color intensity reflects Mkx and Scx expression within each cluster. (B) Box plot of Scx expression in C4_MSCs. Ratio (\%) and number of cells expressing Scx in C4_MSCs is shown at top of box plots. (C) Volcano plot of genes that are differentially expressed between C2_MSCs and C4_MSCs. Differentially expressed genes in C2_MSCs and C4_MSCs. Red and blue circles respectively indicate significantly upregulated genes in C2_MSCs and C4_MSCs. (D) Violin plot of representative significantly upregulated or downregulated genes in C2_MSCs and C4_MSCs.

TABLE 1 | Number and proportion of cells expressing Mkx and SCx in mesenchymal cell clusters.

\begin{tabular}{|c|c|c|c|c|c|}
\hline Cluster ID & Genotype & Total Cell No & $\mathrm{Mkx}^{+}(\%)$ & $\operatorname{Scx}^{+}(\%)$ & $\mathrm{Mkx}^{+} / \mathrm{Scx}^{+}(\%)$ \\
\hline \multirow[t]{2}{*}{ C1_MSCs } & $M k x^{+/+}$ & 3,107 & $331(10.6)$ & $151(4.86)$ & $21(0.6)$ \\
\hline & $M k x^{-/-}$ & 2,920 & $181(6.2)$ & $54(1.8)$ & $4(0.1)$ \\
\hline \multirow[t]{2}{*}{ C2_MSCs } & $M k x^{+/+}$ & 1975 & $331(16.8)$ & $207(10.5)$ & $38(1.9)$ \\
\hline & $M k x^{-/-}$ & 2,688 & $174(6.5)$ & $92(3.5)$ & $6(0.2)$ \\
\hline \multirow[t]{2}{*}{ C4_MSCs } & $M k x^{+/+}$ & 961 & $80(8.3)$ & $178(18.5)$ & $11(1.1)$ \\
\hline & $M k x^{-/-}$ & 1818 & 107 (5.9) & $364(20.0)$ & $26(1.4)$ \\
\hline \multirow[t]{2}{*}{ C6_MSCs } & $M k x^{+/+}$ & 942 & $129(13.7)$ & $68(7.2)$ & $12(1.2)$ \\
\hline & $M k x^{-/-}$ & 413 & $31(7.5)$ & $34(8.2)$ & $1(0.2)$ \\
\hline
\end{tabular}




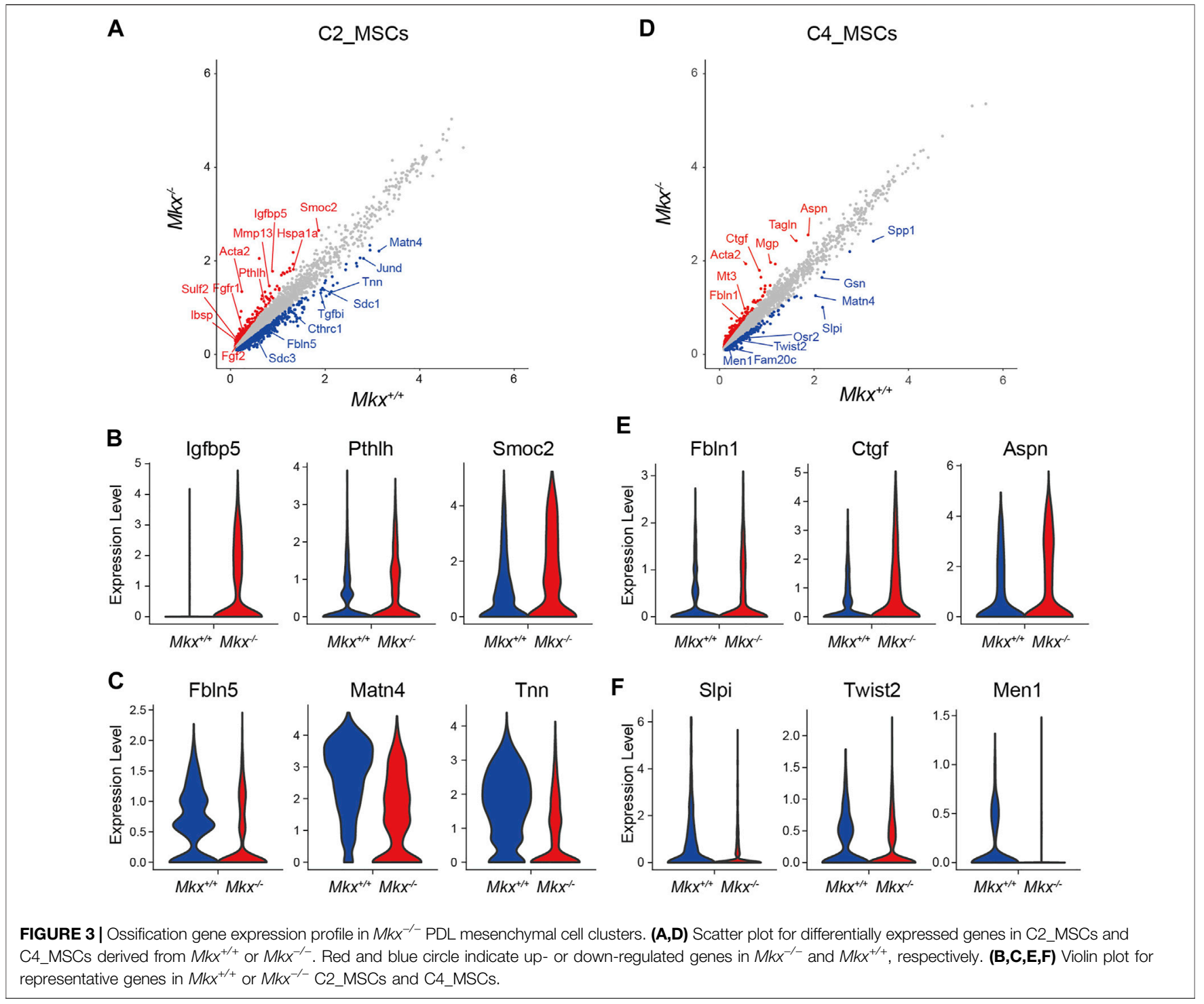

Binding Protein 5, (Igfbp5), bone sialoprotein (Ibsp), SPARC-related modular calcium-binding protein 2 (Smoc2), Osteoprotegerin (Tbfrsf11b), heat shock protein family A member la (Hspala), and Hspalb was upregulated. The number of cells expressing Igfbp5, Pthlh and Smoc2 was remarkably increased in $M k x^{-1-}$ C2_MSCs (Figure 3B). We also identified 1,226 genes with decreased expression, including fibulin5 (Fbnl5), matrilin-4 (Matn4), and Tnn, which are involved in the formation and maintenance of PDL fibers, in $M k x^{-1-}$ C2_MSCs (Figure 3C). In contrast, we found that the expression of 646 genes was upregulated in the C4_MSC cluster. (Figure 3D). The expression of Connective tissue growth factor (Ctgf), which promotes the proliferation of periodontal ligament fibroblasts and collagen production, Asporin (Aspn), which inhibits the ossification of periodontal ligament tissue, and Fibulin extracellular matrix protein 1 (Fbnl1), which is involved in the formation and maintenance of periodontal ligament fibers, were upregulated in $M k x^{-/-}$C4_MSCs (Figure 3E). Gene ontology analysis of 339 downregulated genes in C4_MSCs enriched the annotations involved in ossification $(\log p=-6.33)$ and regulation of biomineral tissue $(\log p=-6.69)$. The number of cells expressing Secretory leukocyte protease inhibitor (Slpi), Twist2 and Menin (Men 1), all of which are important for osteoblast differentiation, was reduced in $\mathrm{Mkx}^{-/-} \mathrm{C} 4$ _MSCs (Figure 3F). These results agreed with our previous findings, and suggested that the loss of $M k x$ promotes ossification in the PDL (Koda et al., 2017). On the other hand, the significant increase in $\mathrm{Scx}^{+}$ cells and the decreased expression of genes involved in ossification in the $\mathrm{Mkx}^{-/-} \mathrm{C} 4$ _MSCs suggested that the loss of $M k x$ promotes compensatory mechanisms to maintain PDL homeostasis via $S c x$. 

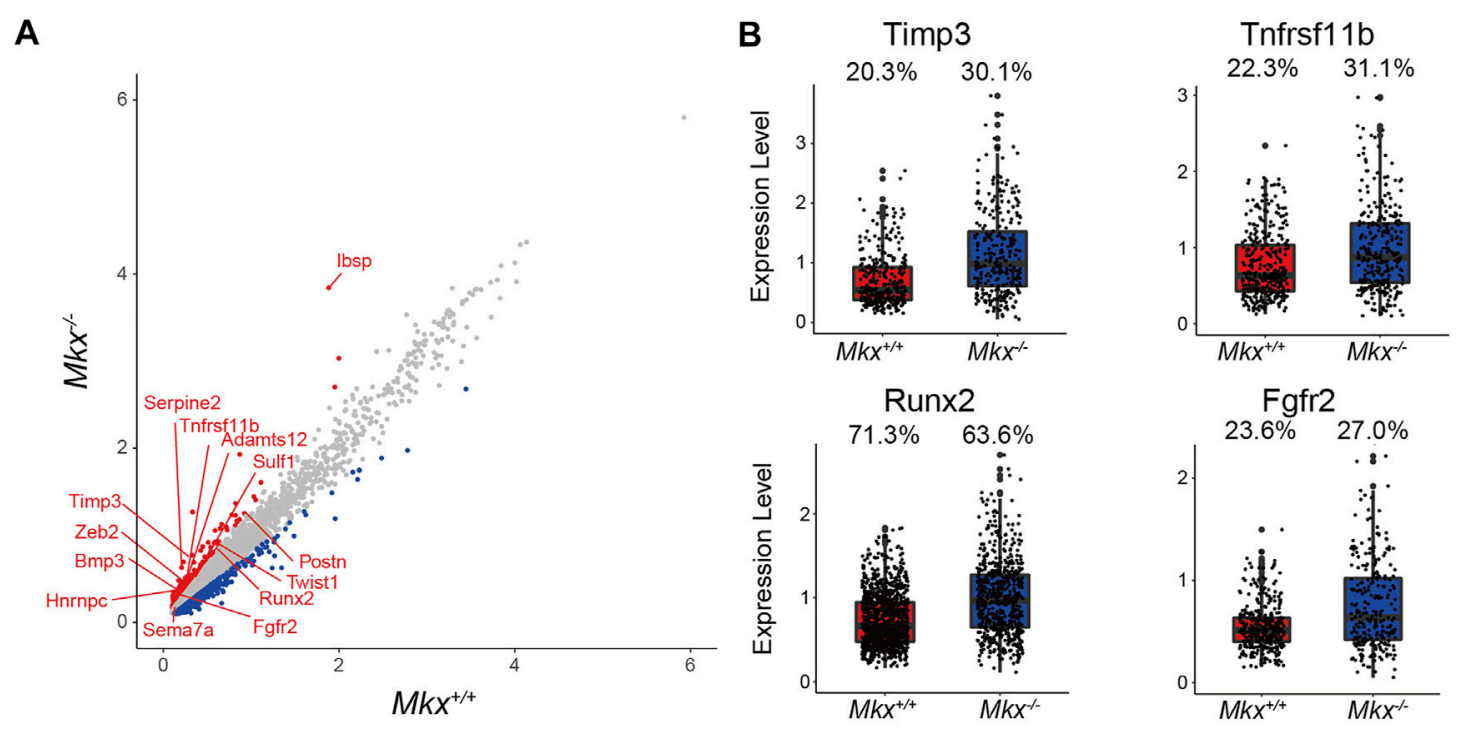

C
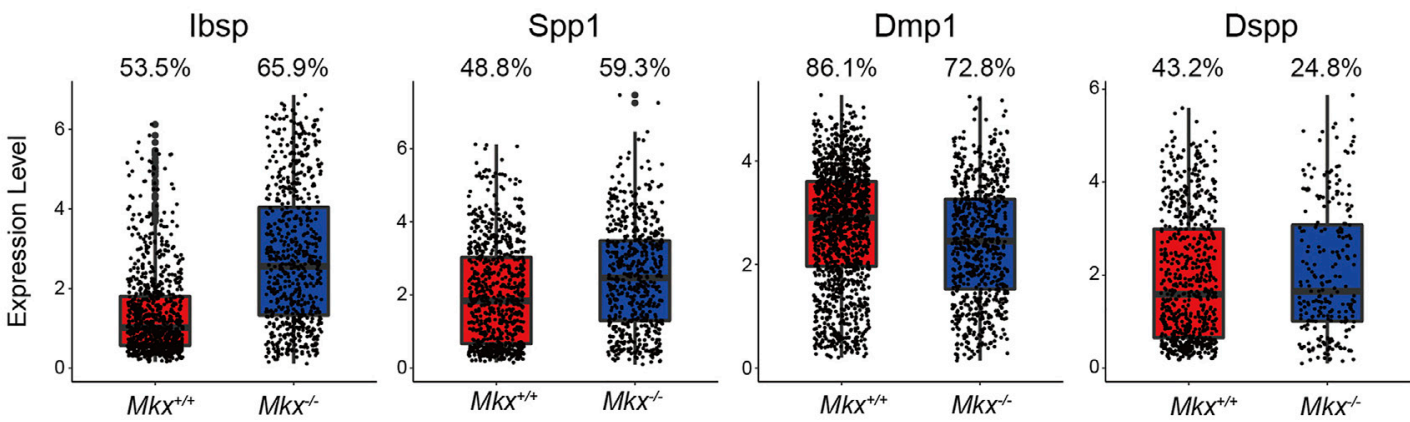

FIGURE 4 | Expression of osteogenic genes is increased in the $M k^{-1 /}$ osteoblast cluster. (A) Scatter plot for differentially expressed genes in C5_OBs derived from $M k x^{+/+}$or $M k^{-1-}$. Red and blue circle indicate up-regulated genes in $M k x^{-/-}$and $M k x^{+/+}$, respectively. (B) Box plots showed the expression of Timp3, Tnfrsf1 1b, Runx2, and Fgfr2 in C5_OBs. (C) The expression of SIBLING family genes in C5_OBs were shown as a box plot. The percentages at the top of the box plots in (B) and (C) indicate the ratio of cells expressing each gene in C5_OBs.

\section{Expression of Osteogenic Genes Is Increased in the $\mathrm{Mkx}^{-/-}$Osteoblast Cluster}

The PDL contains osteoblasts that originate from mesenchymal cells (Basdra and Komposch, 1997; Kovacs et al., 2021). C5_OBs expressed more Nestin and Osteocalcin (Bglap), which are expressed in progenitor and mature osteoblasts (Figure 1B). The expression of Serpine2, Timp3, Tfnrsf11b, Zeb2, Twist1, Ibsp, Sulf1, Postn, Adamts12, Bmp3, Runx2, Tnn, Fgfr2, and Pthlh genes, all of which are annotated with "osteoblast development," was increased in C5_OBs clusters from $M k^{-/-}$ PDL (Figure 4A). The proportion of cells expressing Tissue inhibitor of metalloproteinase 3 (Timp3), Tnfrsf11b, Runx2 and Fgfr2 and expression were increased in the C5_OBs cluster (Figure 4B). In addition, the number of cells expressing the SIBLING family genes Ibsp and Osteopontin (Spp1), but not Dentin matrix acidic phosphoprotein 1 $(D m p 1)$ and Dentin sialophosphoprotein $(D s p p)$, was increased in $\mathrm{Mkx}^{-/-}$osteoblasts within PDL (Figure 4C). These results suggest that the differentiation of osteoblasts, which are involved in the formation of alveolar bone, is enhanced in $\mathrm{Mkx}^{-/-} \mathrm{PDL}$.

\section{Enamel Formation Related Gene Expression Is Promoted in $\mathbf{M k x}^{-/-}$Epithelial Cell Clusters}

Ameloblasts, which produce enamel, arise from epithelial cells (Kovacs et al., 2021). We identified two epithelial cell populations in PDL (Figures 1A,B). The C3_Epi cluster expressed Odam, and the number of cells expressing Amelotin (Amtn), Ameloblastin $(A m b n)$, and Secretory calcium-binding phosphoprotein prolineglutamine rich 1 (Scpppq1), which are involved in enamel formation, were significantly increased in $\mathrm{Mkx}^{-/-}$PDL. Furthermore, the expression of these genes was also increased (Figure 5). These results suggest that enamel formation was enhanced in the $\mathrm{Mkx}^{-/-}$PDL.

\section{Macrophage Clusters With Inflammatory Gene Expression are Larger in Mkx-/- PDL} The C7_Mac cluster contained significantly more cells in PDL from $M k x^{-/-}$, than $M k x^{+/+}$PDL (883 vs. 316 cells). Expression of the pro-inflammatory cytokine Tumor necrosis factor alpha 

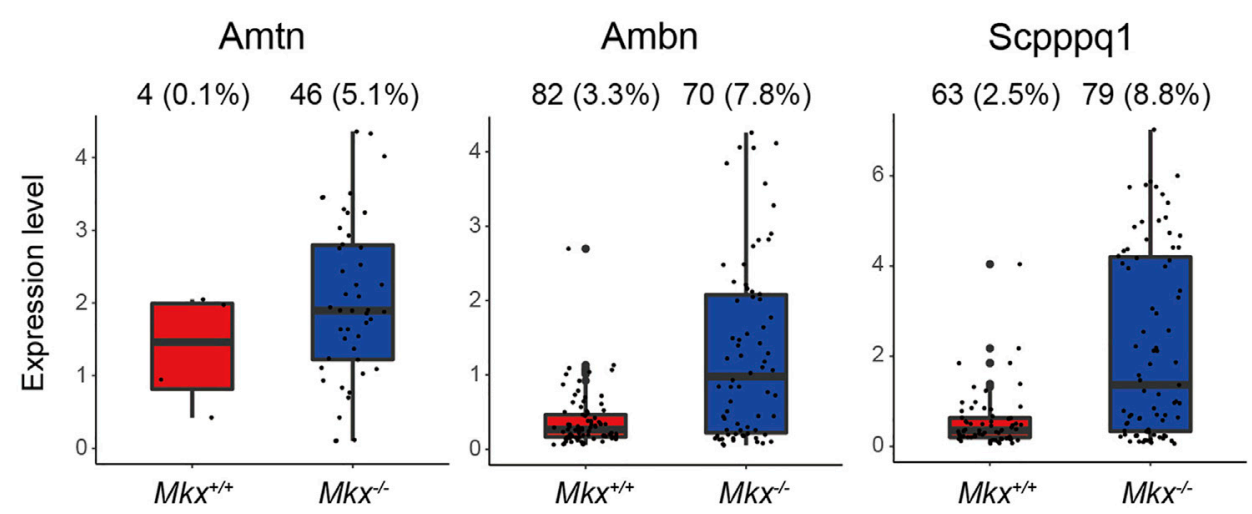

FIGURE 5 | Expression of genes associated with enamel formation are promoted in $\mathrm{Mkx}^{-/-}$epithelial cell clusters. Box plot of Amtn, Ambn, and Scpppq1 expression in C3_Epi. Ratio (\%) of cells expressing each gene in C3_Epi is shown at top of box plots.

$(\operatorname{Tnf})$ and inducible nitric oxide synthase (Nos2) was increased in macrophages from $\mathrm{Mkx}^{-/-} \mathrm{PDL}$ (Figure 6A). The expression of enzymes involved in PKC signaling, such as diacylglycerol synthase Dgat2, phospholipase Pla2g7, and receptors that activate $\mathrm{PKC}$, such as Clec4e, Clec7a, and Trem1, was also upregulated in $\mathrm{Mkx}^{-/-}$macrophages (Figure 6B). The expression of Tribbles pseudokinase 1 (Trib1), Mannose receptor C type 1 (Mrc1), Selenoprotein P (Sepp1), CCAAT/enhancer-binding protein beta (Cebpb), Interleukin 4 receptor (Il4r) V-maf musculoaponeurotic fibrosarcoma oncogene homolog B (Mafb) and Interleukin 10 (Il10) all of which are specifically expressed and/or play important roles in M2 macrophages involved in tissue repair, was also upregulated in macrophages from $\mathrm{Mkx}^{-/-}$PDL (Figure 6C and Supplementary Figure S1). Cell populations expressed Nos2 emerged in $\mathrm{Mkx}^{-/-} \mathrm{PDL}$ whereas such cells were almost undetectable in $\mathrm{Mkx}^{+/+}$ PDL. Unlike macrophages, these cells were negative for CD14, CD68, MHC class II, and TNFa, whereas Mast cellexpressed membrane protein 1 (Mcemp1) and Leucine-rich alpha-2-glycoprotein $(\operatorname{Lrg} 1)$ were detected, suggesting that these populations might be mast cells (Figure 6D and Supplementary Figure S2). We investigated the effects of macrophage-initiated inflammatory responses on other clusters using Pscan that identifies transcription factor binding elements in the promoter regions of genes with variable expression (Zambelli et al., 2009). We found that the increased expression of genes in $\mathrm{Mkx}^{-1-} \mathrm{C} 2$ _MSCs and C4_MSCs was regulated by transcription factors induced by inflammation, such as STAT, AP-1/ATF and NF- $\kappa$ B (Figure 6E). In support of these findings, the expression of Matrix metalloprotein 13 (Mmp13), which is induced by NF$\kappa \mathrm{B}$, was upregulated in mesenchymal cell clusters, whereas the expression of Slpi, which is suppressed by inflammation (Vincenti and Brinckerhoff, 2002; Svensson et al., 2017), was decreased (Figure 6F). These results suggested that increased inflammatory gene expression in macrophages triggers an inflammatory response in other cell populations.

\section{DISCUSSION}

Initially, $M k x$ and $S c x$ were described as transcription factors involved in tendon development (Cserjesi et al., 1995; Ito et al., 2010; Liu et al., 2010; Schweitzer et al., 2001), and they play important roles in the development and homeostasis of tendon cells. The PDL, like tendon cells, is a fibrous tissue composed mainly of type 1 collagen, and $M k x$ and $S c x$ are important for the development and homeostasis of PDL (Koda et al., 2017; Miyazaki et al., 2021; Takimoto et al., 2015). Here, we identified mesenchymal cell populations expressing $M k x$ and $S c x$ in PDL using scRNA-Seq. Cells expressing $M k x$ and $S c x$ were abundant in C2_MSCs and C4_MSCs, respectively. Few cells expressed both $M k x$ and $S c x$ among the mesenchymal stromal cells (Table 1). It had been reported that $S c x$ was expressed mainly in fibroblasts located in the central part of the PDL by using Scx-GFP reporter mice (Takimoto et al., 2015). In contrast, $M k x$ expression was seen throughout the PDL in Mkx-Venus reporter mice (Koda et al., 2017). The present study using scRNA-Seq revealed that $M k x$ and Scx expressions are mutually exclusive, which suggests that cells expressing $M k x$ and $S c x$ are localized to different regions in the PDL and play different roles in PDL development and homeostasis.

We previously showed that $M k x$ maintains and enhances the expression of type 1 and type 3 collagen in tendons and PDL, and that its loss leads to progressive ossification of the tendons and PDL (Koda et al., 2017; Miyazaki et al., 2021; Suzuki et al., 2016). The present study found upregulated expression of the Igfbp5, Pthlh, HSP70 family genes (Hspala and Hspa1b), and SIBLING family gene Ibsp, all of which are important for osteogenesis, in

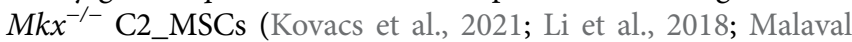
et al., 2008; Miyakoshi et al., 2001). On the other hand, the expression of Fbln5 and Matn4, which are involved in the formation of oxytalan fibers within the PDL, was decreased in the $\mathrm{Mkx}^{-1-}$ C2_MSCs (Figure 3C) (Hisanaga et al., 2009; Schubert et al., 2017). The expression of Tnn, which is important for formation of the extracellular matrix of the PDL, was also decreased in $M k x^{-/-}$C2_MSCs (Imhof et al., 
A

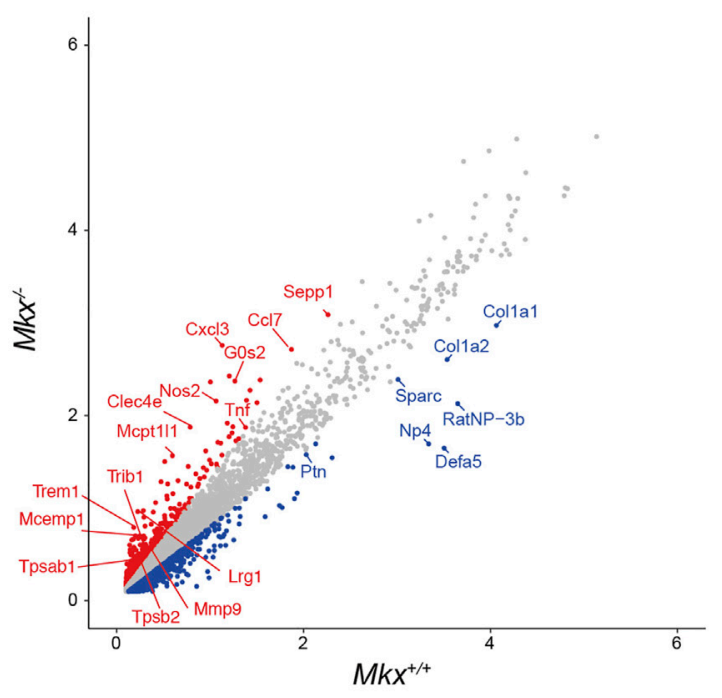

B

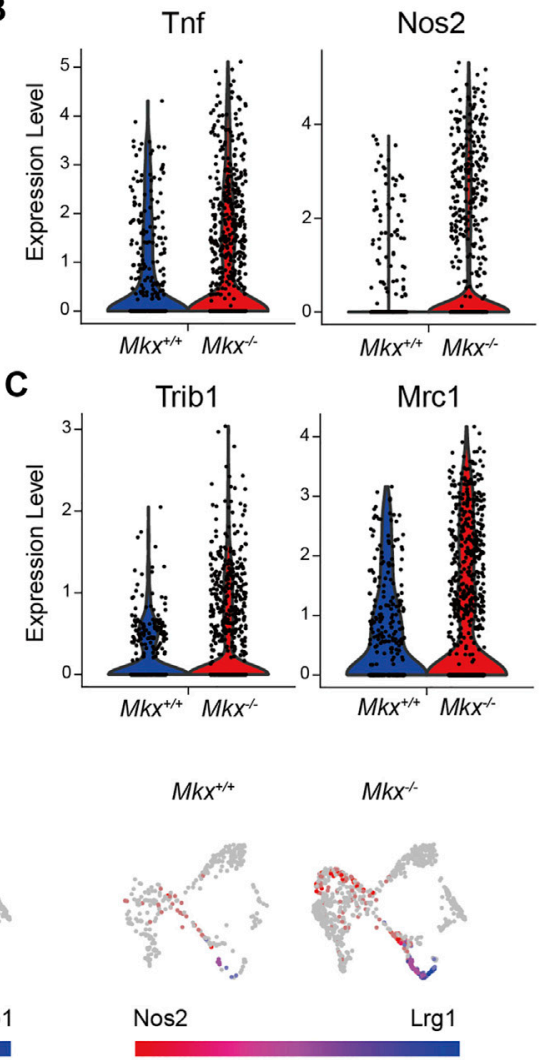

E
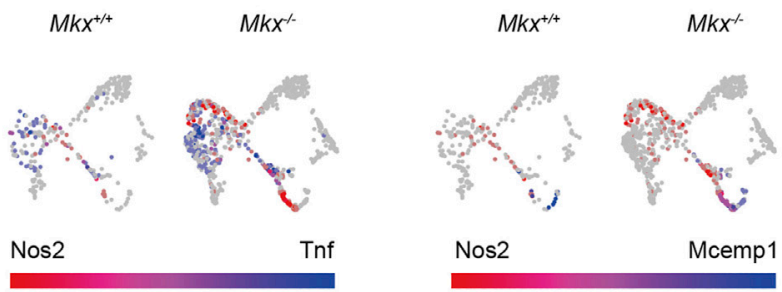

F

$M k x^{+\alpha+} \square M k x^{\prime-}$

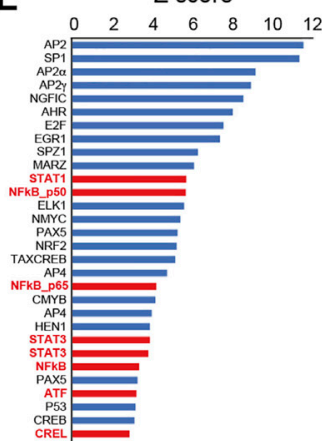

C2_MSCs

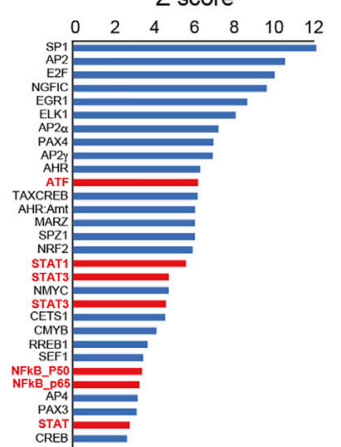

C4_MSCs

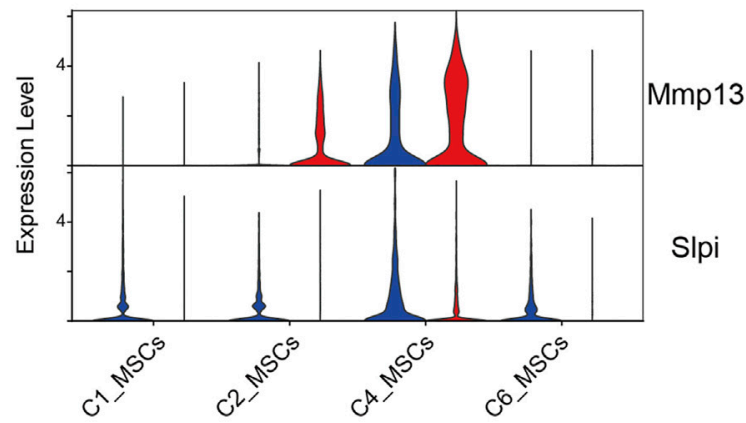

FIGURE 6 | Macrophages cluster with inflammatory gene expressions increases in Mkx ${ }^{-/}$PDL. (A) Scatter plot for differentially expressed genes in C7_Mac derived from $\mathrm{MkX}^{+/+}$or $\mathrm{Mkx}^{-/-}$. Red and blue circle indicate up-regulated genes in $\mathrm{Mkx}^{-/-}$and $\mathrm{Mkx}^{+/+}$, respectively. (B,C) Box plot for the expression of Tnf, Nos2, Trib1, and Mrc1 in C7 Mac. (D) Co-expression of Nos2 with Tnf, Mcemp1, and Lrg1 in C7 Mac. The color intensity reflects the co-expression level within each cluster (E) Pscan analysis revealed that genes with increased expression in C2_MSCs and C4_MSCs. The top 30 transcription factors involved in the regulation of gene expression in C2_MSCs and C4_MSCs were shown. Transcription factors involved in inflammation were shown in red. (F) Violin plot for the expression of Mmp13 and Slpi in C1, C2, C4, and C6_MSCs.

2020). These results suggested that $M k x$ promotes the production of various extracellular matrices and their regulatory molecules, which are important for the formation of PDL fibers and inhibit PDL ossification. Notably, Scx-positive cells were significantly more prevalent in $\mathrm{Mkx}^{-/-} \mathrm{C} 4$ _MSCs. This was supported by the increased expression of $F b \ln 1$, which is involved in the formation of oxytalan fibers, connective tissue growth factor (Ctgf), which enhances the proliferation of periodontal ligament cells and type 1 collagen, Periostin, and Aspn that inhibit the ossification of mesenchymal cells (Asano et al., 2005; Yamada et al., 2007; Schubert et al., 2017). Scx prevents remodeling of the periodontal tissues induced by osterix-induced ossification under tensile force (Takimoto et al., 2015). Although the inhibition of ossification by $S c x$ is a cell-intrinsic mechanism 
and the effect on $M k x$-expressing cells is not clear, these results suggest compensatory role(s) of $S c x$ in $M k x^{-/-}$PDL.

The C5_OBs cluster in $M k x^{-/-}$PDL contained fewer cells. However, the number of cells expressing Runx2, which plays a central role in osteoblast differentiation, and Timp3, Tnfrsf $11 \mathrm{~b}$ and Fgfr2, which are important for osteoblast differentiation, inhibition of cell death, and proliferation, were increased in C5_OBs (Javaheri et al., 2016; Kovacs et al., 2021; Simonet et al., 1997; Su et al., 2014). The number of cells expressing Ibsp, which belongs to the bone SIBLING protein family, and the amount of Ibsp expression were significantly increased. The number of cells expressing Osteopontin (Spp1) was also slightly increased. These results suggested that the loss of $M k x$ enhances bone formation by osteoblasts. However, although both osteoblasts and cells expressing $M k x$ are derived from mesenchymal cells, it is unclear whether an $M k x$ deficiency directly converts them to osteoblasts by increasing their plasticity or indirectly enhances the differentiation and activity of osteoblasts (Basdra and Komposch, 1997; Kovacs et al., 2021). Therefore, further analysis is needed to elucidate the inhibitory roles of $M k x$ in PDL ossification.

Enamel is an inorganic tissue that is almost devoid of cells, and ameloblasts, enamel matrix-producing cells, are derived from odontogenic epithelial cells (Kovacs et al., 2021). The Odontogenic ameloblast-associated protein (Odam), that is abundantly expressed in the C3_Epi, generates enamel matrix by forming complexes with Amelotin (Amtn), Ameloblastin (Ambn), and the Secreted calcium-binding protein Scpppq1 (Fouillen et al., 2017). The number of cells expressing Amtn, Ambn, and Scpppq1 are increased in $\mathrm{Mkx}^{-/-}$C3_Epi. The PDL contains epithelial cells called epithelial rests of Malassez (ERM). However, the physiological significance of increased Amtn, Ambn, and Scpppq1 expression remains obscure because enamel does not form in adult rat molars. In contrast, the expression of Ambn, Amtn, and Odam is upregulated in ERM during root repair (Hasegawa et al., 2003; Nishio et al., 2010). Since the activation of ERM is important for cementum repair and regeneration of the width of PDL, the loss of Mkx might lead to ERM activation due to a fragile PDL and increased inflammatory response (Lindskog et al., 1988; Hasegawa et al., 2003; Keinan and Cohen, 2013). However, since the expression of these genes is also upregulated in gingival epithelial cells, that this is a contamination of gingival epithelial cells cannot be ruled out and requires further detailed investigation (Nakayama et al., 2017).

The finding of more clusters containing macrophages with increased Tnf and Nos2 expression in $M k x^{-/-}$, than $M k x^{+/+}$PDLs indicated enhancement of the inflammatory response. Macrophages can differentiate into functionally distinct macrophages with different roles in innate immune responses and tissue repair (Murray and Wynn, 2011; Italiani and Borachi., 2014). M1 macrophages are activated by bacterial lipopolysaccharide and IFN $\gamma$ to produce abundant inflammatory mediators and they have a superior capacity for antigen presentation (Murray and Wynn, 2011; Italiani and Borachi., 2014). Unlike M1 macrophages, M2 macrophages are activated by IL- 4 and IL-13, and they have less capacity to produce inflammatory mediators and presenting antigens but have more capacity for tissue regeneration
(Murray and Wynn, 2011; Italiani and Borachi., 2014). The expression of Sepp1 and Mrc1, which play important roles in M2 macrophages was increased in $M \mathrm{kx}^{-/-}$rats, along with Trib1, Cebpb, $I l 4 r$, Illo and Mafb, which are involved in M2 macrophage differentiation (Ruffell et al., 2009; Gordon and Martinez, 2010; Satoh et al., 2013; Barrett et al., 2015; Kim 2017). These results suggested that robust inflammation was induced in $\mathrm{Mkx}^{-/-} \mathrm{PDL}$, followed by M2 macrophage differentiation and/or mobilization.

In addition to inflammatory triggers generated by oral bacteria, PDL inflammation is controlled by mechanical loading. (Chukkapalli and Lele, 2018). We previously showed that the loss of $M k x$ in tendons and ligaments, including the PDL, results in hypoplastic tendon and ligament formation and a consequent loss of tendon and ligament function (Ito et al., 2010; Suzuki et al., 2016; Koda et al., 2017; Miyazaki et al., 2021). The present study found upregulated ossification-related genes and downregulated genes involved in the formation of PDL in $M k x^{-/-}$PDL. These results suggested that hypoplastic PDL formation could induce increased mechanical loading in $\mathrm{Mkx}^{-1-} \mathrm{PDL}$, leading to an increased inflammatory response.

We detected Nos2-positive cells in $M k^{-1-}$ C7_Mac that did not express macrophage markers, but expressed Lrg1 and Mcemp1, assuming that they are mast cells. Mast cells induce periodontal inflammation by producing not only allergic mediators but also various inflammatory mediators, including nitric oxide (Sheethal et al., 2018). Activation of mast cells by inflammatory cytokines and ligands for pattern-recognition receptors result in the production of MMPs and Tryptase, which promote degradation of the ECM (Sheethal et al., 2018; Checchi et al., 2020). In fact, we found that the expression of $M m p 8$, Tpsab1, and Tpsb2 that are involved in collagen degradation, was increased in C7_Mac cluster. These results suggested that macrophage activation induces production of ECM-degrading enzymes from mast cells and macrophages themself, leading to hypoplastic PDL fibers in $M k x^{-1-}$ rat. Forced expression of $M k x$ in meniscus cells in knee joints negatively regulates inflammation by inhibiting the expression of ADAMTS-5, IL-6, MMP1, and MMP3, suggesting that $M k x$ itself is involved in the regulation of inflammation (Lee et al., 2020). The intrinsic role of $M k x$ in inflammation awaits clarification.

In conclusion, the expression of $M k x$ and $S c x$ was mutually exclusive, suggesting that they play different roles in the development and maintenance of PDL. In addition, the expression of genes involved in ossification in mesenchymal cells and osteoblasts and the inflammatory responses of macrophages was increased in $M \mathrm{kx}^{-/-}$PDLs, which could lead to hypoplastic PDL formation. These results suggest that $M k x$ is involved in ankylosis and periodontitis, and thus further detailed investigation is required.

\section{DATA AVAILABILITY STATEMENT}

The datasets presented in this study can be found in online repositories. The names of the repository/repositories and accession number(s) can be found below: DRA012879 and DRA013311 in DDBJ (https://ddbj.nig.ac.jp/DRASearch/). 


\section{ETHICS STATEMENT}

The animal study was reviewed and approved by the Institutional Animal Care and Use Committee of the Tokyo Medical and Dental University. Written informed consent was obtained from the owners for the participation of their animals in this study.

\section{AUTHOR CONTRIBUTIONS}

KT performed experiment. TC interpreted data and wrote a manuscript. TM, LY, RN, TI, KM, and HH provided a critical insight into this study. HA supervised for this study. All authors read the final version of the manuscript and agreed to its submission.

\section{FUNDING}

This work was supported by the Japan Society for the Promotion of Science KAKENHI (Grant Nos. 20H05696 and 18K19603 to

\section{REFERENCES}

Asano, M., Kubota, S., Nakanishi, T., Nishida, T., Yamaai, T., Yosimichi, G., et al. (2005). Effect of Connective Tissue Growth Factor (CCN2/CTGF) on Proliferation and Differentiation of Mouse Periodontal Ligament-Derived Cells. Cell Commun. Signal. 3, 11. doi:10.1186/1478-811X-3-11

Barrett, C. W., Reddy, V. K., Short, S. P., Motley, A. K., Lintel, M. K., Bradley, A. M., et al. (2015). Selenoprotein P Influences Colitis-Induced Tumorigenesis by Mediating Stemness and Oxidative Damage. J. Clin. Invest. 125, 2646-2660. doi:10.1172/JCI76099

Basdra, E. K., and Komposch, G. (1997). Osteoblast-like Properties of Human Periodontal Ligament Cells: an In Vitro Analysis. Eur. J. Orthod. 19, 615-621. doi:10.1093/ejo/19.6.615

Beertsen, W., McCulloch, C. A. G., and Sodek, J. (1997). The Periodontal Ligament: a Unique, Multifunctional Connective Tissue. Periodontol. 2000 13, 20-40. doi:10.1111/j.1600-0757.1997.tb00094.x

Butler, A., Hoffman, P., Smibert, P., Papalexi, E., and Satija, R. (2018). Integrating Single-Cell Transcriptomic Data across Different Conditions, Technologies, and Species. Nat. Biotechnol. 36, 411-420. doi:10.1038/nbt.4096

Checchi, V., Maravic, T., Bellini, P., Generali, L., Consolo, U., Breschi, L., et al. (2020). The Role of Matrix Metalloproteinases in Periodontal Disease. Ijerph 17, 4923. doi:10.3390/ijerph17144923

Chen, Y., Guan, Q., Han, X., Bai, D., Li, D., and Tian, Y. (2021). Proteoglycans in the Periodontium: A Review with Emphasis on Specific Distributions, Functions, and Potential Applications. J. Periodont Res. 56, 617-632. doi:10. $1111 /$ jre. 12847

Chiba, Y., Saito, K., Martin, D., Boger, E. T., Rhodes, C., Yoshizaki, K., et al. (2020). Single-Cell RNA-Sequencing from Mouse Incisor Reveals Dental Epithelial Cell-type Specific Genes. Front. Cel Dev. Biol. 8, 841. doi:10.3389/fcell.2020. 00841

Chukkapalli, S. S., and Lele, T. P. (2018). Periodontal Cell Mechanotransduction. Open Biol. 8, 180053. doi:10.1098/rsob.180053

Cserjesi, P., Brown, D., Ligon, K. L., Lyons, G. E., Copeland, N. G., Gilbert, D. J., et al. (1995). Scleraxis: a Basic helix-loop-helix Protein that Prefigures Skeletal Formation during Mouse Embryogenesis. Development 121, 1099-1110. doi:10. 1242/dev.121.4.1099

Fouillen, A., Dos Santos Neves, J., Mary, C., Castonguay, J.-D., Moffatt, P., Baron, C., et al. (2017). Interactions of AMTN, ODAM and SCPPPQ1 Proteins of a
HA), NIH grant (AR050631 to HA), and AMED-CREST from AMED (Grant No. JP21gm0810008 to HA).

\section{ACKNOWLEDGMENTS}

We thank all of member of Department of Systems BioMedicine at Tokyo Medical and Dental University for their helpful discussion.

\section{SUPPLEMENTARY MATERIAL}

The Supplementary Material for this article can be found online at: https://www.frontiersin.org/articles/10.3389/fcell.2022.795441/ full\#supplementary-material

Supplementary Figure S1 | Expression of PKC signaling-related and M2 macrophage-related genes in macrophage clusters. (A,B) Violin plot shows expression of Dgat2, Pla2g7, Clec4e, Clec7a, Trem1, Mafb, Sepp1, Cebpb, IIr, and $\| 10$ in C7_Mac.

Supplementary Figure $\mathbf{5 2}$ | Expression of macrophage marker genes in macrophage clusters. Expression of macrophage marker genes Cd14 and Cd68, MHC class II (RT1-Db1), and Lyz2 in C7_Mac.

Specialized Basal Lamina that Attaches Epithelial Cells to Tooth mineral. Sci. Rep. 7, 46683. doi:10.1038/srep46683

Franco, C., Patricia, H.-R., Timo, S., Claudia, B., and Marcela, H. (2017). Matrix Metalloproteinases as Regulators of Periodontal Inflammation. Ijms 18, 440. doi:10.3390/ijms 18020440

Gordon, S., and Martinez, F. O. (2010). Alternative Activation of Macrophages: Mechanism and Functions. Immunity 32, 593-604. doi:10.1016/j.immuni.2010. 05.007

Hajishengallis, G., and Chavakis, T. (2021). Local and Systemic Mechanisms Linking Periodontal Disease and Inflammatory Comorbidities. Nat. Rev. Immunol. 21, 426-440. doi:10.1038/s41577-020-00488-6

Harrel, S. K. (2003). Occlusal Forces as a Risk Factor for Periodontal Disease. Periodontol. 2000 32, 111-117. doi:10.1046/j.0906-6713.2002. 03209.x

Hasegawa, N., Kawaguchi, H., Ogawa, T., Uchida, T., and Kurihara, H. (2003). Immunohistochemical Characteristics of Epithelial Cell Rests of Malassez during Cementum Repair. J. Periodontal Res. 38, 51-56. doi:10.1034/j.16000765.2003.01636.x

Hisanaga, Y., Nakashima, K., Tsuruga, E., Nakatomi, Y., Hatakeyama, Y., Ishikawa, H., et al. (2009). Fibulin-5 Contributes to Microfibril Assembly in Human Periodontal Ligament Cells. Acta Histochem. Cytochem. 42, 151-157. doi:10. 1267/ahc.09021

Holt, S. C., and Ebersole, J. L. (2005). Porphyromonas Gingivalis, Treponema Denticola, and Tannerella Forsythia: the 'red Complex', a Prototype Polybacterial Pathogenic Consortium in Periodontitis. Periodontol. 2000 38, 72-122. doi:10.1111/j.1600-0757.2005.00113.x

Imhof, T., Balic, A., Heilig, J., Chiquet-Ehrismann, R., Chiquet, M., Niehoff, A., et al. (2020). Pivotal Role of Tenascin-W (-N) in Postnatal Incisor Growth and Periodontal Ligament Remodeling. Front. Immunol. 11, 608223. doi:10.3389/ fimmu.2020.608223

Ishikawa, I., Iwata, T., Washio, K., Okano, T., Nagasawa, T., Iwasaki, K., et al. (2009). Cell Sheet Engineering and Other Novel Cell-Based Approaches to Periodontal Regeneration. Periodontol. 2000 51, 220-238. doi:10.1111/j.16000757.2009.00312.x

Italiani, P., and Boraschi, D. (2014). From Monocytes to M1/M2 Macrophages: Phenotypical vs. Functional Differentiation. Front. Immunol. 5, 514. doi:10. 3389/fimmu.2014.00514

Ito, Y., Toriuchi, N., Yoshitaka, T., Ueno-Kudoh, H., Sato, T., Yokoyama, S., et al. (2010). The Mohawk Homeobox Gene Is a Critical Regulator of Tendon 
Differentiation. Proc. Natl. Acad. Sci. 107, 10538-10542. doi:10.1073/pnas. 1000525107

Javaheri, B., Hopkinson, M., Poulet, B., Pollard, A. S., Shefelbine, S. J., Chang, Y.M., et al. (2016). Deficiency and Also Transgenic Overexpression of Timp-3 Both Lead to Compromised Bone Mass and Architecture In Vivo. PLoS One 11, e0159657. doi:10.1371/journal.pone.0159657

Keinan, D., and Cohen, R. E. (2013). The Significance of Epithelial Rests of Malassez in the Periodontal Ligament. J. Endodontics 39, 582-587. doi:10.1016/ j.joen.2013.01.004

Kim, H. (2017). The Transcription Factor MafB Promotes Anti-inflammatory M2 Polarization and Cholesterol Efflux in Macrophages. Sci. Rep. 7, 7591. doi:10. 1038/s41598-017-07381-8

Kinane, D. F., Stathopoulou, P. G., and Papapanou, P. N. (2017). Periodontal Diseases. Nat. Rev. Dis. Primers 3, 17038. doi:10.1038/nrdp.2017.38

Koda, N., Sato, T., Shinohara, M., Ichinose, S., Ito, Y., Nakamichi, R., et al. (2017). Mohawk Transcription Factor Regulates Homeostasis of the Periodontal Ligament. Development 144, 313-320. doi:10.1242/dev.135798

Kovacs, C. S., Chaussain, C., Osdoby, P., Brandi, M. L., Clarke, B., and Thakker, R. V. (2021). The Role of Biomineralization in Disorders of Skeletal Development and Tooth Formation. Nat. Rev. Endocrinol. 17, 336-349. doi:10.1038/s41574021-00488-z

Krivanek, J., Soldatov, R. A., Kastriti, M. E., Chontorotzea, T., Herdina, A. N., Petersen, J., et al. (2020). Dental Cell Type Atlas Reveals Stem and Differentiated Cell Types in Mouse and Human Teeth. Nat. Commun. 11, 4816. doi:10.1038/s41467-020-18512-7

Lalla, E., and Papapanou, P. N. (2011). Diabetes Mellitus and Periodontitis: a Tale of Two Common Interrelated Diseases. Nat. Rev. Endocrinol. 7, 738-748. doi:10.1038/nrendo.2011.106

Lamont, R. J., and Hajishengallis, G. (2015). Polymicrobial Synergy and Dysbiosis in Inflammatory Disease. Trends Mol. Med. 21, 172-183. doi:10.1016/j.molmed. 2014.11.004

Lee, K. I., Gamini, R., Olmer, M., Ikuta, Y., Hasei, J., Baek, J., et al. (2020). Mohawk Is a Transcription Factor that Promotes Meniscus Cell Phenotype and Tissue Repair and Reduces Osteoarthritis Severity. Sci. Transl. Med. 12. doi:10.1126/ scitranslmed.aan7967

Lerner, U. H. (2006). Inflammation-induced Bone Remodeling in Periodontal Disease and the Influence of post-menopausal Osteoporosis. J. Dent. Res. 85, 596-607. doi:10.1177/154405910608500704

Li, C., Sunderic, K., Nicoll, S. B., and Wang, S. (2018). Downregulation of Heat Shock Protein 70 Impairs Osteogenic and Chondrogenic Differentiation in Human Mesenchymal Stem Cells. Sci. Rep. 8, 553. doi:10.1038/s41598-01718541-1

Lindskog, S., Blomlof, L., and Hammarstrom, L. (1988). Evidence for a Role of Odontogenic Epithelium in Maintaining the Periodontal Space. J. Clin. Periodontol. 15, 371-373. doi:10.1111/j.1600-051x.1988.tb01014.x

Liu, W., Watson, S. S., Lan, Y., Keene, D. R., Ovitt, C. E., Liu, H., et al. (2010). The Atypical Homeodomain Transcription Factor Mohawk Controls Tendon Morphogenesis. Mol. Cel. Biol. 30, 4797-4807. doi:10.1128/MCB.00207-10

Malaval, L., Wade-Gue'ye, N. M., Boudiffa, M., Fei, J., Zirngibl, R., Chen, F., et al. (2008). Bone Sialoprotein Plays a Functional Role in Bone Formation and Osteoclastogenesis. J. Exp. Med. 205, 1145-1153. doi:10.1084/jem.20071294

McKee, M. D., Hoac, B., Addison, W. N., Barros, N. M. T., Millán, J. L., and Chaussain, C. (20132000). Extracellular Matrix Mineralization in Periodontal Tissues: Noncollagenous Matrix Proteins, Enzymes, and Relationship to Hypophosphatasia and X-Linked Hypophosphatemia. Periodontol. 2000 63, 102-122. doi:10.1111/prd.12029

Miyakoshi, N., Richman, C., Kasukawa, Y., Linkhart, T. A., Baylink, D. J., and Mohan, S. (2001). Evidence that IGF-Binding Protein-5 Functions as a Growth Factor. J. Clin. Invest. 107, 73-81. doi:10.1172/JCI10459

Miyazaki, T., Kurimoto, R., Chiba, T., Matsushima, T., Nakamichi, R., Tsutsumi, H., et al. (2021). Mkx Regulates the Orthodontic Tooth Movement via Osteoclast Induction. J. Bone Miner. Metab. 39, 780-786. doi:10.1007/ s00774-021-01233-2

Murray, P. J., and Wynn, T. A. (2011). Protective and Pathogenic Functions of Macrophage Subsets. Nat. Rev. Immunol. 11, 723-737. doi:10.1038/nri3073

Nakayama, Y., Kobayashi, R., Matsui, S., Matsumura, H., Iwai, Y., Noda, K., et al. (2017). Localization and Expression Pattern of Amelotin, Odontogenic Ameloblast-Associated Protein and Follicular Dendritic Cell-Secreted
Protein in the Junctional Epithelium of Inflamed Gingiva. Odontology 105, 329-337. doi:10.1007/s10266-016-0277-y

Nilsson, B. O. (2021). Mechanisms Involved in Regulation of Periodontal Ligament Cell Production of Pro-inflammatory Cytokines: Implications in Periodontitis. J. Periodont Res. 56, 249-255. doi:10.1111/jre.12823

Nishio, C., Wazen, R., Kuroda, S., Moffatt, P., and Nanci, A. (2010). Disruption of Periodontal Integrity Induces Expression of Apin by Epithelial Cell Rests of Malassez. J. Periodontal Res. 45, 709-713. doi:10.1111/j.1600-0765.2010. 01288.x

Pagella, P., de Vargas Roditi, L., Stadlinger, B., Moor, A. E., and Mitsiadis, T. A. (2021). A Single-Cell Atlas of Human Teeth. iScience 24, 102405. doi:10.1016/j. isci.2021.102405

Ruffell, D., Mourkioti, F., Gambardella, A., Kirstetter, P., Lopez, R. G., Rosenthal, N., et al. (2009). A CREB-C/EBP cascade Induces M2 Macrophage-specific Gene Expression and Promotes Muscle Injury Repair. Proc. Natl. Acad. Sci. 106, 17475-17480. doi:10.1073/pnas.0908641106

Satoh, T., Kidoya, H., Naito, H., Yamamoto, M., Takemura, N., Nakagawa, K., et al. (2013). Critical Role of Trib1 in Differentiation of Tissue-Resident M2-like Macrophages. Nature 495, 524-528. doi:10.1038/nature11930

Schubert, A., Schminke, B., and Miosge, N. (2017). Fibulins and Matrilins Are Novel Structural Components of the Periodontium in the Mouse. Arch. Oral Biol. 82, 216-222. doi:10.1016/j.archoralbio.2017.06.008

Schweitzer, R., Chyung, J. H., Murtaugh, L. C., Brent, A. E., Rosen, V., Olson, E. N., et al. (2001). Analysis of the Tendon Cell Fate Using Scleraxis, a Specific Marker for Tendons and Ligaments. Development 128, 3855-3866. doi:10.1242/dev. 128.19 .3855

Sheethal, H. S., Kn, H., Smitha, T., and Chauhan, K. (2018). Role of Mast Cells in Inflammatory and Reactive Pathologies of Pulp, Periapical Area and Periodontium. J. Oral Maxillofac. Pathol. 22, 92-97. doi:10.4103/jomfp. JOMFP_278_17

Simonet, W. S., Lacey, D. L., Dunstan, C. R., Kelley, M., Chang, M.-S., Lüthy, R., et al. (1997). Osteoprotegerin: a Novel Secreted Protein Involved in the Regulation of Bone Density. Cell 89, 309-319. doi:10.1016/s0092-8674(00) 80209-3

Socransky, S. S., and Haffajee, A. D. (20052000). Periodontal Microbial Ecology. Periodontol. 2000 38, 135-187. doi:10.1111/j.1600-0757.2005.00107.x

Strydom, H., Maltha, J. C., Kuijpers-Jagtman, A. M., and Von den Hoff, J. W. (2012). The Oxytalan Fibre Network in the Periodontium and its Possible Mechanical Function. Arch. Oral Biol. 57, 1003-1011. doi:10.1016/j. archoralbio.2012.06.003

Su, N., Jin, M., and Chen, L. (2014). Role of FGF/FGFR Signaling in Skeletal Development and Homeostasis: Learning from Mouse Models. Bone Res. 2, 14003. doi:10.1038/boneres.2014.3

Suzuki, H., Ito, Y., Shinohara, M., Yamashita, S., Ichinose, S., Kishida, A., et al. (2016). Gene Targeting of the Transcription Factor Mohawk in Rats Causes Heterotopic Ossification of Achilles Tendon via Failed Tenogenesis. Proc. Natl. Acad. Sci. USA 113, 7840-7845. doi:10.1073/pnas.1522054113

Svensson, D., Aidoukovitch, A., Anders, E., Jönsson, D., Nebel, D., and Nilsson, B.O. (2017). Secretory Leukocyte Protease Inhibitor Regulates Human Periodontal Ligament Cell Production of Pro-inflammatory Cytokines. Inflamm. Res. 66, 823-831. doi:10.1007/s00011-017-1062-2

Takahashi, A., Nagata, M., Gupta, A., Matsushita, Y., Yamaguchi, T., Mizuhashi, K., et al. (2019). Autocrine Regulation of Mesenchymal Progenitor Cell Fates Orchestrates Tooth Eruption. Proc. Natl. Acad. Sci. USA 116, 575-580. doi:10. 1073/pnas. 1810200115

Takimoto, A., Kawatsu, M., Yoshimoto, Y., Kawamoto, T., Seiryu, M., TakanoYamamoto, T., et al. (2015). Scleraxis and Osterix Antagonistically Regulate Tensile Force-Responsive Remodeling of the Periodontal Ligament and Alveolar Bone. Development 142, 787-796. doi:10.1242/dev.116228

Tanay, A., and Regev, A. (2017). Scaling Single-Cell Genomics from Phenomenology to Mechanism. Nature 541, 331-338. doi:10.1038/nature21350

Vincenti, M. P., and Brinckerhoff, C. E. (2002). Transcriptional Regulation of Collagenase (MMP-1, MMP-13) Genes in Arthritis: Integration of Complex Signaling Pathways for the Recruitment of Gene-specific Transcription Factors. Arthritis Res. Ther. 4, 157-164. doi:10.1186/ar401

Yamada, S., Tomoeda, M., Ozawa, Y., Yoneda, S., Terashima, Y., Ikezawa, K., et al. (2007). PLAP-1/asporin, a Novel Negative Regulator of Periodontal Ligament Mineralization. J. Biol. Chem. 282, 23070-23080. doi:10.1074/jbc.M611181200 
Zambelli, F., Pesole, G., and Pavesi, G. (2009). Pscan: Finding Over-represented Transcription Factor Binding Site Motifs in Sequences from Co-regulated or Co-expressed Genes. Nucleic Acids Res. 37, W247-W252. doi:10.1093/nar/ gkp464

Conflict of Interest: The authors declare that the research was conducted in the absence of any commercial or financial relationships that could be construed as a potential conflict of interest.

Publisher's Note: All claims expressed in this article are solely those of the authors and do not necessarily represent those of their affiliated organizations, or those of the publisher, the editors and the reviewers. Any product that may be evaluated in this article, or claim that may be made by its manufacturer, is not guaranteed or endorsed by the publisher.

Copyright (c) 2022 Takada, Chiba, Miyazaki, Yagasaki, Nakamichi, Iwata, Moriyama, Harada and Asahara. This is an open-access article distributed under the terms of the Creative Commons Attribution License (CC BY). The use, distribution or reproduction in other forums is permitted, provided the original author(s) and the copyright owner(s) are credited and that the original publication in this journal is cited, in accordance with accepted academic practice. No use, distribution or reproduction is permitted which does not comply with these terms. 